\title{
Radiation Transport in Type IA Supernovae
}

\author{
R. G. Eastman
}

This article was submitted to

NATO Astro Physics Conference, Aiguablava, Spain, June 20-30, 1995

\section{November 16, 1999}

U.S. Department of Energy

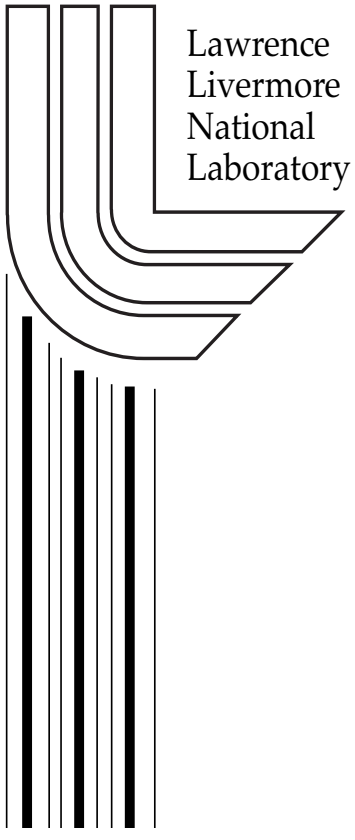




\section{DISCLAIMER}

This document was prepared as an account of work sponsored by an agency of the United States Government. Neither the United States Government nor the University of California nor any of their employees, makes any warranty, express or implied, or assumes any legal liability or responsibility for the accuracy, completeness, or usefulness of any information, apparatus, product, or process disclosed, or represents that its use would not infringe privately owned rights. Reference herein to any specific commercial product, process, or service by trade name, trademark, manufacturer, or otherwise, does not necessarily constitute or imply its endorsement, recommendation, or favoring by the United States Government or the University of California. The views and opinions of authors expressed herein do not necessarily state or reflect those of the United States Government or the University of California, and shall not be used for advertising or product endorsement purposes.

This is a preprint of a paper intended for publication in a journal or proceedings. Since changes may be made before publication, this preprint is made available with the understanding that it will not be cited or reproduced without the permission of the author.

This report has been reproduced

directly from the best available copy.

Available to DOE and DOE contractors from the

Office of Scientific and Technical Information

P.O. Box 62, Oak Ridge, TN 37831

Prices available from (423) 576-8401

http: / / apollo.osti.gov/ bridge/

Available to the public from the

National Technical Information Service

U.S. Department of Commerce

5285 Port Royal Rd., Springfield, VA 22161

http:/ / www.ntis.gov/

OR

Lawrence Livermore National Laboratory

Technical Information Department's Digital Library

http: / / www.llnl.gov/tid/Library.html 


\title{
RADIATION TRANSPORT IN TYPE IA SUPERNOVAE
}

\author{
RONALD G. EASTMAN \\ Lawrence Livermore National Laboratory \\ Livermore, CA, USA 94550
}

\section{Introduction}

It has been said more than once that the critical link between explosion models and observations is the ability to accurately simulate cooling and radiation transport in the expanding ejecta of Type Ia supernovae. It is perhaps frustrating to some of the theorists who study explosion mechanisms, and to some of the observers too, that more definitive conclusions have not been reached about the agreement, or lack thereof, between various Type Ia supernova models and the data. Although claims of superlative accuracy in transport simulations are sometimes made, I will argue here that there are outstanding issues of critical importance and in need of addressing before radiation transport calculations are accurate enough to discriminate between subtly different explosion models.

\section{Opacity in Type Ia Supernovae}

For calculating the evolution of a Type Ia supernova, it is only a slight exaggeration to say that opacity is everything. As others have stressed (Harkness 1991; Wheeler, Swartz and Harkness 1993), the ability to determine the correct bolometric rise time and maximum luminosity, as well as other observable quantities of interest, depends as sensitively on knowing the opacity as on the total mass of the star. For conditions relevant to SNe Ia around maximum light, the opacity at optical wavelengths is predominately electron scattering. However, in the ultraviolet it is dominated by a thick forest of lines whose opacity effect is increased by Doppler broadening (Karp et al. 1977).

Several sources of uncertainty exist regarding the line blanketing opacity. One involves the atomic data. The line list most commonly employed is that of Kurucz (1991). Because the number of lines which contribute to the 
total opacity is so large, numbering in the hundreds of thousands or more, there is no way at present to know either how accurate or how complete the list is with respect to the weaker lines. As Harkness (1991) has stressed, the bulk of the opacity comes from the cumulative effect of many weak lines of iron group elements, and the lack of any information about completeness of the data set must be regarded as an unknown source of uncertainty in the calculations.

The number of transitions is too large to be precisely represented on a frequency grid of reasonable size, and approximations to its representation must employed to keep the grid size at a tractable level. One such approximation is that proposed by Karp et al. (1977), and employed by Höflich and his collaborators (Höflich, Müller \& Khokhlov 1993). Another is that proposed by Eastman \& Pinto (1993; also Pinto, this volume), which bears some similarity to the ideas of Wagoner, Perez and Vasu (1991). Blinnikov (this volume) discusses the shortcomings of the Karp opacity and proposes a more accurate replacement. The approximate line opacity expression devised by Eastman and Pinto considers the statistical effect of many lines on the underlying continuum, but it underestimates the opacity of strong, isolated transitions.

Figure 1 shows the wavelength dependent opacity contributions in a mixture of ${ }^{56} \mathrm{Ni}(20 \%),{ }^{56} \mathrm{Co}(70 \%)$ and ${ }^{56} \mathrm{Fe}(10 \%)$, at a density of $\rho=$ $10^{-13} \mathrm{~g} \mathrm{~cm}^{-3}, T=2.5 \times 10^{4} \mathrm{~K}$ and $t=14$ days after explosion, assuming the excitation and ionization are given by the Saha-Boltzmann equation and the line opacity approximation of Eastman and Pinto (1993). In this case, the optical opacity is dominated by electron scattering, followed by free-free, and in the UV, by lines. As Montes and Wagoner (1995) have pointed out, between 2000 and $4000 \AA$ the wavelength dependence of the line opacity goes like $d \ln \kappa_{\lambda} / d \ln \lambda \sim-10$. As explained below, the steep wavelength dependence has important consequences for frequency integrated mean opacities. Near maximum light, the central temperature of SNe la ranges from $2 \times 10^{4} \mathrm{~K}$ to $3 \times 10^{4} \mathrm{~K}$. At $2.5 \times 10^{4} \mathrm{~K}$, the peak of the Planck function is near $2000 \AA$, where the optical depth due to lines is very high. Radiation deposited in the interior scatters around until it has either been Doppler shifted far enough into the red that it can random walk its way out on a short time scale, or more likely, branches into several longer wavelength photons which are able to escape.

\subsection{FREQUENCY INTEGRATED MEAN OPACITIES}

The full blown, time dependent, frequency dependent, non-LTE radiation transport problem is an expensive calculation, in terms of the large amounts of memory and cpu cycles required. A commonly employed expedient is to 
RADIATION TRANSPORT IN TYPE IA SUPERNOVAE
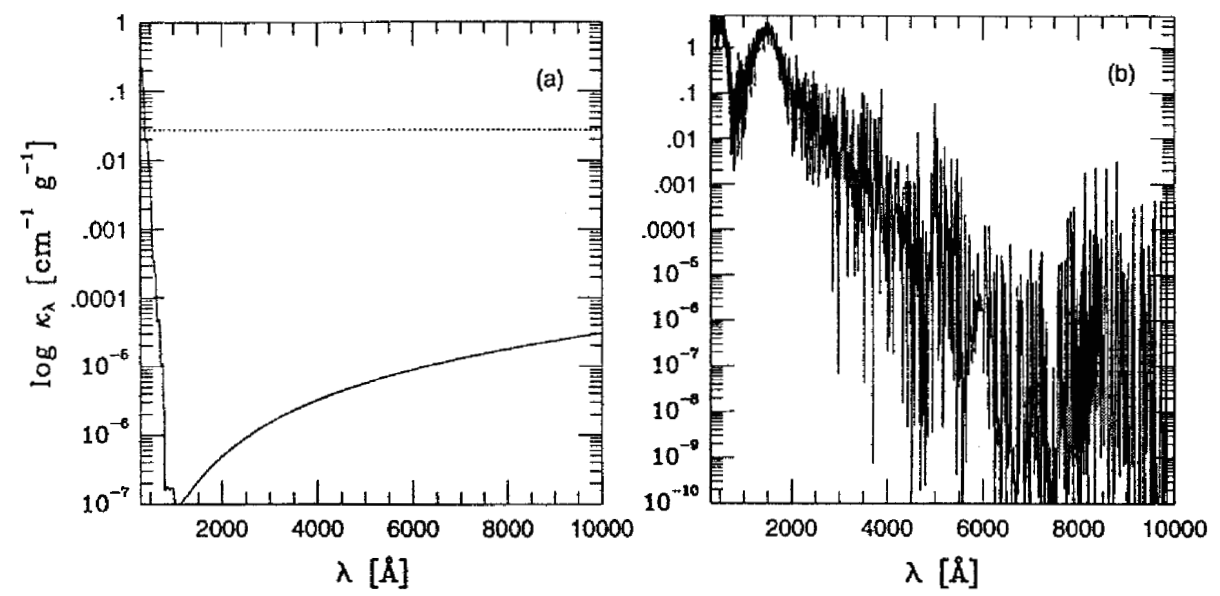

Figure 1. This figure shows the contributions to the total opacity for a gas consisting of $70 \% \mathrm{Ni}, 20 \% \mathrm{Co}$ and $10 \% \mathrm{Fe}$, at a density of $10^{-13} \mathrm{~g} \mathrm{~cm}^{-3}$ and temperature of $25,000 \mathrm{~K}$, with populations given by the Saha-Boltzmann equation. In (a) is plotted the mass opacity coefficient for electron scattering (dotted line) and the sum of bound-free and free-free (solid line). In (b) is plotted the mass opacity coefficient of lines in the expansion opacity approximation of Eastman \& Pinto (1993), assuming homologous expansion and $t=14$ days.

integrate the transport equation over frequency and solve a single frequency integrated transport equation where the opacity terms have been replaced with appropriate means. The most important of these is the flux mean, defined as

$$
\kappa_{H}=\int_{0}^{\infty} \kappa_{\nu} H_{\nu} d \nu / H
$$

where

$$
H \equiv \int_{0}^{\infty} H_{\nu} d \nu
$$

is the bolometric flux. If $H_{\nu}$ is known, which generally requires already knowing the solution, then $\kappa_{H}$ can be computed. Usually it is not known, so another approximation is made, called the diffusion approximation, in which the angular distribution of the radiation field is assumed isotropic, the time rate of change of $H_{\nu}$ is ignored, and $H_{\nu}$ is specified in terms of $\partial J_{\nu} / \partial r$, where $J_{\nu}$ is the radiation field mean intensity. If it is further assumed that $J_{\nu}=B_{\nu}(T)=$ the Planck function, this gives rise to the Rosseland mean opacity, $\kappa_{R}$. Blinnikov (this volume) gives a detailed discussion of the Rosseland mean opacity and how it is modified by expansion. In $\S 3.3$ below I present the results of a comparison between $\kappa_{H}$ and $\kappa_{R}$ obtained from a multi-group transfer solution. 


\section{RONALD G. EASTMAN}

All published evolutionary calculations have so far been based on the assumption that the gas is in local thermodynamic equilibrium (LTE), in which excitation and ionization are given by the Saha-Boltzmann equation at the local gas temperature. There are only two ways by which this can be brought about: one is if the electron density is high enough that collisions drive the level populations to a Boltzmann distribution. The other is if the optical depth is high and the distance over which a photon travels before thermal destruction is much shorter than the characteristic length over which gas temperature changes. Clearly this condition does not hold at the photosphere, which by definition is the place at which photons escape to infinity. In $\S 3.1 \mathrm{I}$ will show that even at maximum light in a Chandrasekhar mass explosion there are significant differences between the radiation field mean intensity $J$ and the local gas temperature Planck function $B$.

\subsection{ABSORPTION VERSUS SCATTERING}

The assumption of LTE raises another serious issue with the line opacity, and that is the question of how much of it is absorptive and how much is purely scattering. Blinnikov et al. (1996) show that in multi-group calculations, substantial variations are obtained in both the bolometric and the broad band light curve of a SN 1993J model depending on whether the line opacity, or some fraction of it, is taken to be scattering or absorptive. This is not surprising, as quanta which can be absorbed have a much greater probability of reappearing at longer wavelengths where the optical depth is lower, which can decrease the random walk escape time by a significant amount. If exit channels involving photoabsorption are ignored, it is straightforward to estimate the relative probabilities of escape from the resonance region, of thermal destruction, and of splitting. For a photon absorbed in a transition between lower level $i$ and upper level $j$, the probability that it will escape from the resonance region is

$$
p_{i j}(\text { escape })=\frac{A_{i j} \beta_{i j}}{\sum_{l} A_{l j} \beta_{l j}+N_{e} \sum_{l} q_{l j}}
$$

where $\beta_{i j}$ is the Sobolev escape probability, $N_{e}$ is the electron density and $q_{i j}$ is the electron collision rate coefficient. Similarly, the probability of thermal destruction by electron collision while in the excited state is

$$
p_{i j}(\text { therm })=\frac{N_{e} \sum_{l} q_{l j}}{\sum_{l} A_{l j} \beta_{l j}+N_{e} \sum_{l} q_{l j}}
$$

while the splitting probability is just

$$
p_{i j}(\text { split })=1-p_{i j}(\text { therm })-p_{i j}(\text { escape })
$$




\section{RADIATION TRANSPORT IN TYPE IA SUPERNOVAE}

For each transition contributing to the line opacity in Figure 1, the probability of scattering (escape from resonance region), thermalization by electron collisional depopulation of the excited level, and splitting, was computed and the opacity contribution divided into scattering, thermal and splitting components by weighting the total opacity with the respective probability. The result are shown in Figure 2, which plots the total weighted line opacity for each process. Except at optical and infrared wavelengths, the probability of thermal destruction is very small. In both the near and far UV, an absorbed photon is 5 to 10 times more likely to be split than scattered. By assuming LTE and taking the line opacity to be absorptive, the effect of branching is crudely taken into account. The situation is rather analogous to static gas transport in a single line with complete redistribution and weak thermal coupling. This is a well studied problem in which the line source function is known to depart significantly from the continuum source function even at large optical depths. Given the results of Blinnikov et al. (1996) and the importance of this process, I predict that issue of Type Ia supernovae and their progenitors will not be fully resolved until the state of the art is multi-group non-LTE radiation transport on iron peak elements.

\section{Multi-Group Transport Calculations}

An alternative to the approach described above of using a Rosseland mean opacity to solve a frequency integrated transport equation, or to the diffusion approximation, is to divide frequency or wavelength space up into bins and compute a solution to the coupled, fully time dependent, monochromatic transport problem. The calculations presented below were performed with a modified version of the code EDDINGTON (Eastman \& Pinto 1993). The calculations assume the velocity field of the explosion model is frozen in and freely expanding, and LTE is also assumed, for which more will be said below.

In EDDINGTON, the gas temperature is determined by solving the time dependent first law of thermodynamics, with energy input by radiative absorption and heating by Compton scattering of $\gamma$-rays, and losses by expansion and radiative emission (because the energy density is dominated by radiation, at maximum light the same gas temperature would be obtained by balancing heating and cooling and ignoring the gas pressure contribution to $P d V$ losses). The local deposition rate from radioactive decay is determined with reasonable accuracy by doing a separate, deterministic transport calculation for each $\gamma$-ray emission line, as described by Woosley et al. (1994).

Blinnikov et al. (1996) obtain excellent agreement in results on test problems run with EDDINGTON and with the multi-group implicit radia- 
RONALD G. EASTMAN

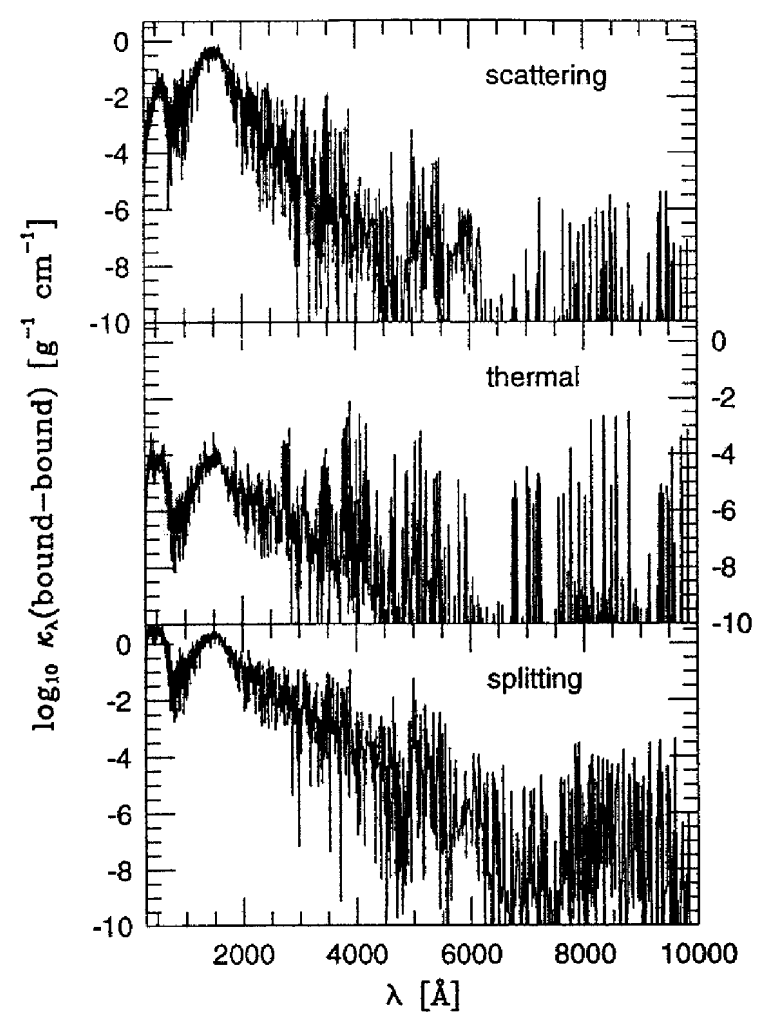

Figure 2. This figure shows the same total line opacity given in the lower panel of Figure 1, except that the opacity of each transition is weighted by the probability of escape from the resonance region as a photon of the same initial wavelength (scattering), of collisional destruction (thermal), and of destruction by downward branching to an alternate level (splitting).

tion hydrodynamics code STELLA (Blinnikov \& Bartunov 1993; Bartunov et al. 1994), when the same approximations are used in both codes for the line opacity. In the SNe Ia model calculations described below, the line opacity was taken to be entirely absorptive, and a variably spaced wavelength grid of 600 points was used ranging from $50 \AA$ to $20 \mu$.

\subsection{THREE TYPE IA SUPERNOVA MODELS}

The following discussion is based on results from multi-group transport calculations on three models: the well studied $M_{c h}$ deflagration Model W7 (Nomoto, Thielemann, \& Sokoi 1984), an $M_{c h}$ delayed detonation Model DD4 (Woosley \& Weaver 1991), and a sub- $M_{c h}$ surface helium detonation model, Model 4 (Woosley \& Weaver 1994). Table 1 summarizes basic prop- 


\section{RADIATION TRANSPORT IN TYPE IA SUPERNOVAE}

erties of the three models.

TABLE 1. Type Ia Explosion Models

\begin{tabular}{lllll}
\hline Model & $\mathrm{M}\left[\mathrm{M}_{\odot}\right]$ & $\mathrm{E}\left[10^{51} \mathrm{ergs} \mathrm{s}^{-1}\right]$ & $\mathrm{M}\left({ }^{56} \mathrm{Ni}\right)\left[\mathrm{M}_{\odot}\right]$ & ref \\
\hline DD4 & 1.39 & 1.22 & 0.63 & Woosley \& Weaver (1992) \\
W7 & 1.22 & 1.17 & 0.63 & Nomoto et al. (1984) \\
Model 2 & 0.90 & 1.06 & 0.43 & Woosley \& Weaver (1994) \\
\hline
\end{tabular}

Figure 3 shows the bolometric light curve for the three models, and compares them to the bolometric light curves of SN 1989B, SN 1992A and SN 1991T which are given by Suntzeff (1995). The distance moduli and reddenings for each of these supernovae was taken from Phillips (1993). Either of the two $M_{c h}$ models is a good fit to $1991 \mathrm{~T}$, and if one accepts the accuracy of the Tully-Fisher distance modulus on which the bolometric data points are based, then these results imply that only the brightest SNe Ia's come from $M_{c h}$ progenitors, while the most common SNe Ia, which are fainter, come from sub- $M_{c h}$ progenitors.

Figures 4 and 5 compare the computed B and V light curves of Model W7 and Model DD4 with the observations of 91T. The agreement is good enough to admit the plausibility of either model. However, concerns over the assumption of LTE make it impossible to ascribe the discrepancies between the model light curve and $91 \mathrm{~T}$ to shortcomings of the explosion model. No homogenization was done on the outer layers of Model W7, as suggested by Harkness (1991).

Figure 6 compares the UBVRI light curves of SN 1989B from Wells et al. (1994) to those of the sub- $M_{c h}$ Model 2. The extinction to $89 \mathrm{~B}$ was taken as $E(B-V)=0.34$ (Wells et al. 1994) and the distance modulus as 29.4 (Phillips 1993). In all 5 bands shown in Figure 6, the agreement at maximum light between the broad band luminosity of $89 \mathrm{~B}$ and of those of Model 2 are quite reasonable, but get progressively worse after maximum light. That fact does not mean that $89 \mathrm{~B}$ was not the explosion of a sub- $M_{c h}$ white dwarf. More likely, what it shows is that as the supernova expands and cools, excitation and ionization conditions become more and more nebular, and light curves based on an LTE calculations cannot be expected to behave identically to the observations.

One thing which Figure 6 shows, albeit in an exaggerated fashion, is the same type of secondary IR maximum which is also seen in the data. It also shows up quite clearly in the calculated bolometric curve (Figure 3 ). The 
RONALD G. EASTMAN

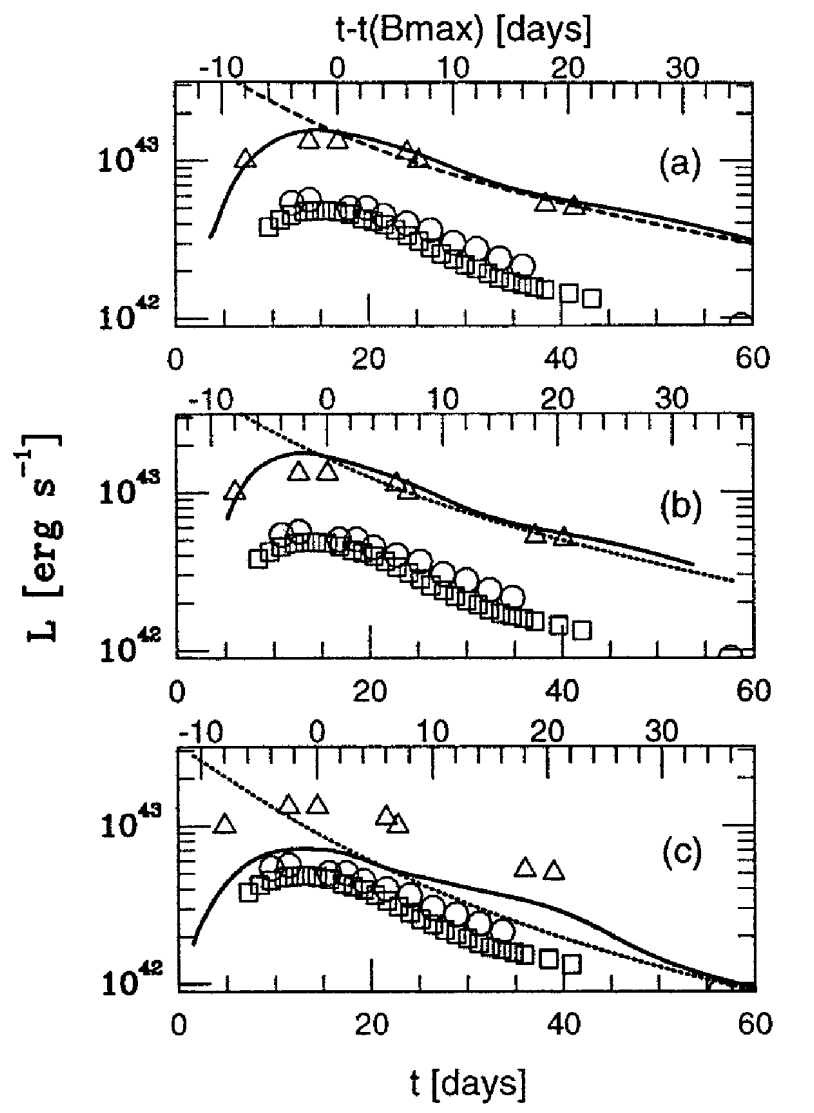

Figure 3. This figure compares the bolometric light curves for the three models listed in Table 1 with the bolometric light curves derived from observations by Suntzeff (1995) for SN 1991 T (triangles), SN 1989B (circles) and SN 1992T (squares). Figure 3a gives the bolometric light curve for Model DD4 (solid line) and the total instantaneous deposition rate (dashed line). Figures $3 \mathrm{~b}$ and c show the same thing, but for Models W7 and Model 2, respectively.

mechanism which causes the secondary maximum is a cooling of the core which shifts the emission to longer wavelengths. Its net effect is similar to the recombination wave which moves through the hydrogen envelopes of Type II supernovae, but it is different because the core remains optically thick to ultraviolet photons. As ionization in the core drops, new channels appear for energy to be absorbed in the ultraviolet and reemitted as optical and near infrared photons. This process further lowers the temperature and ionization, and a short lived thermal instability ensues which last only until the core has stabilized at a lower temperature and ionization state. The secondary maximum which observationally is most prominent in the $R$ and 
RADIATION TRANSPORT IN TYPE IA SUPERNOVAE

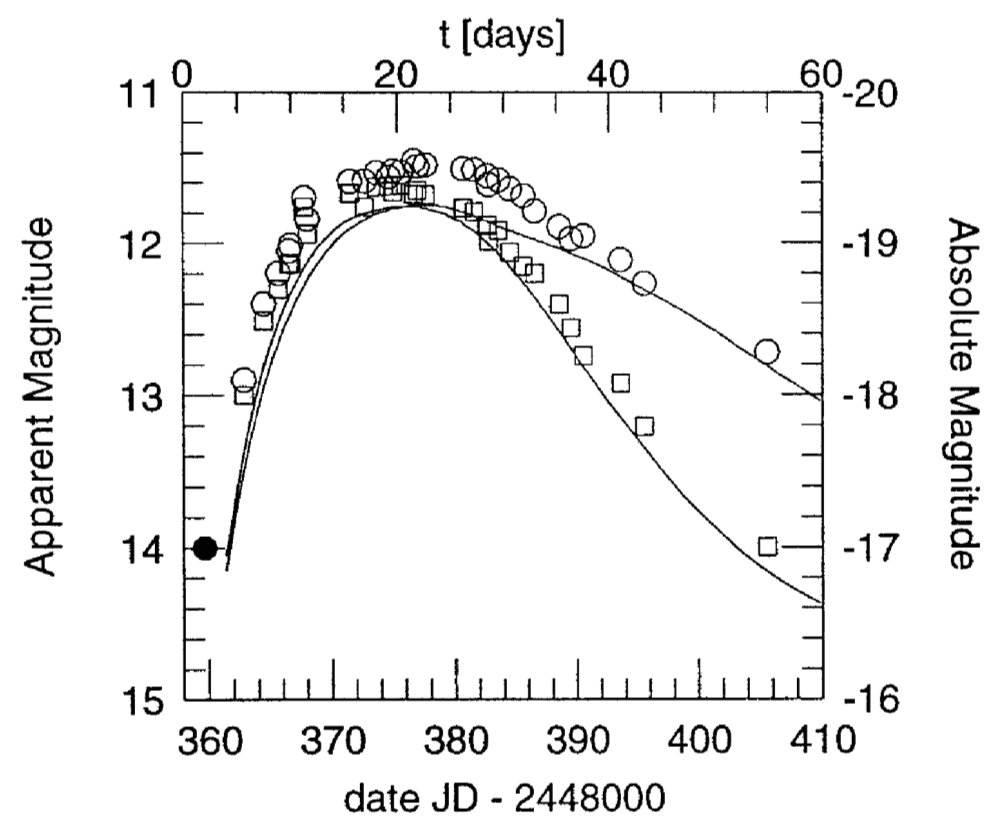

Figure 4. This figure compares B (squares) and V (circles) photometry of SN 1991T (Phillips et al. 1992) to the calculated B and V light curve of Model DD4.

$I$ bands, is powered by stored energy released by this thermal instability.

\subsection{OPTICAL DEPTHS AND PHOTON THERMALIZATION}

As discussed previously, LTE is a good approximation only when the electron density is large, or when the photon thermal destruction length is small. Figures 7 and 8 show the total optical depth versus wavelength to the center, as measured in the observer frame, for Model DD4 and of Model 2, respectively, at 14 days after explosion. These figures also show the thermalization optical depth, which is defined as

$$
\tau_{\lambda}(\text { therm })=\int_{0}^{R_{\max }} \sqrt{k_{\lambda}\left(k_{\lambda}+\sigma_{\lambda}\right)} d R
$$

where $k_{\lambda}$ and $\sigma_{\lambda}$ are the absorptive and scattering opacities, respectively, in the observer frame. At 14 days the electron scattering optical depth in Model DD4 is $\sim 30$, but the thermalization depth is, at several places in the optical and near infrared, as small as $\sim 2$. In Model 2 the electron scattering optical depth at 14 days is $\sim 5$, and the thermalization optical depth even smaller, in some windows as small as 0.2 . The actual path of an optical photon as it diffuses outward is more convoluted than a straight line 


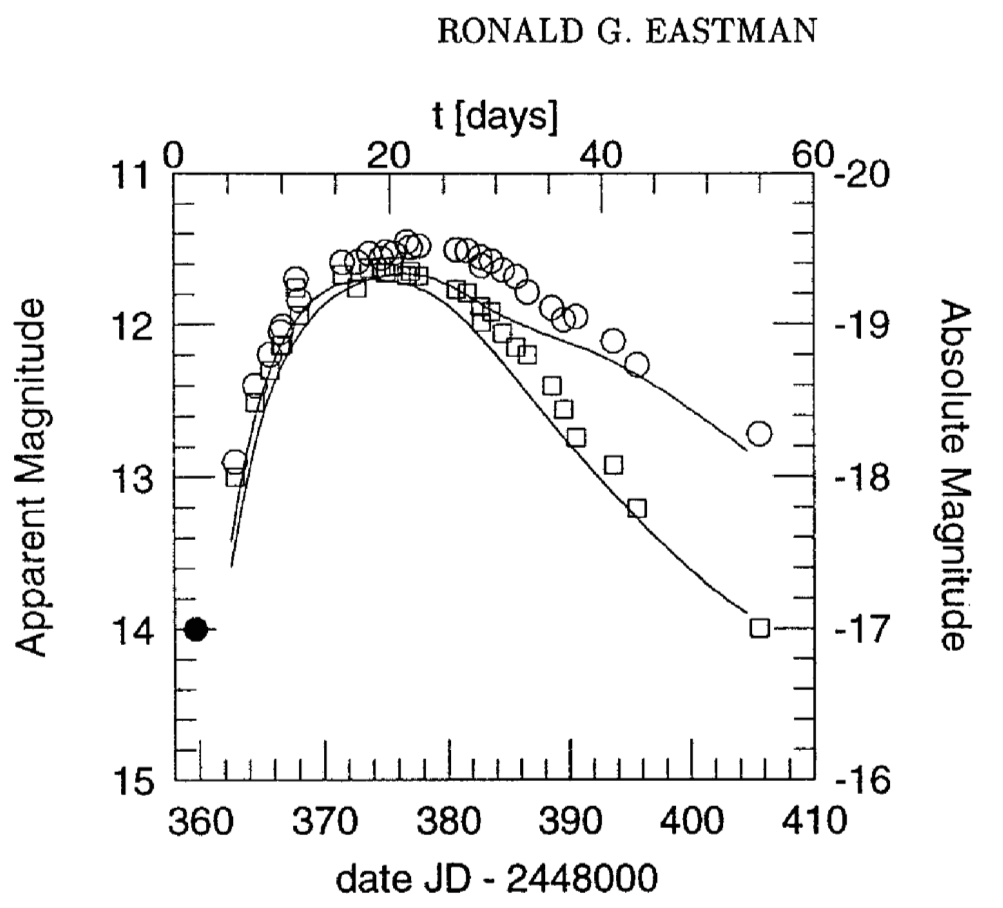

Figure 5. Same as Figure 4, but for Model W7.

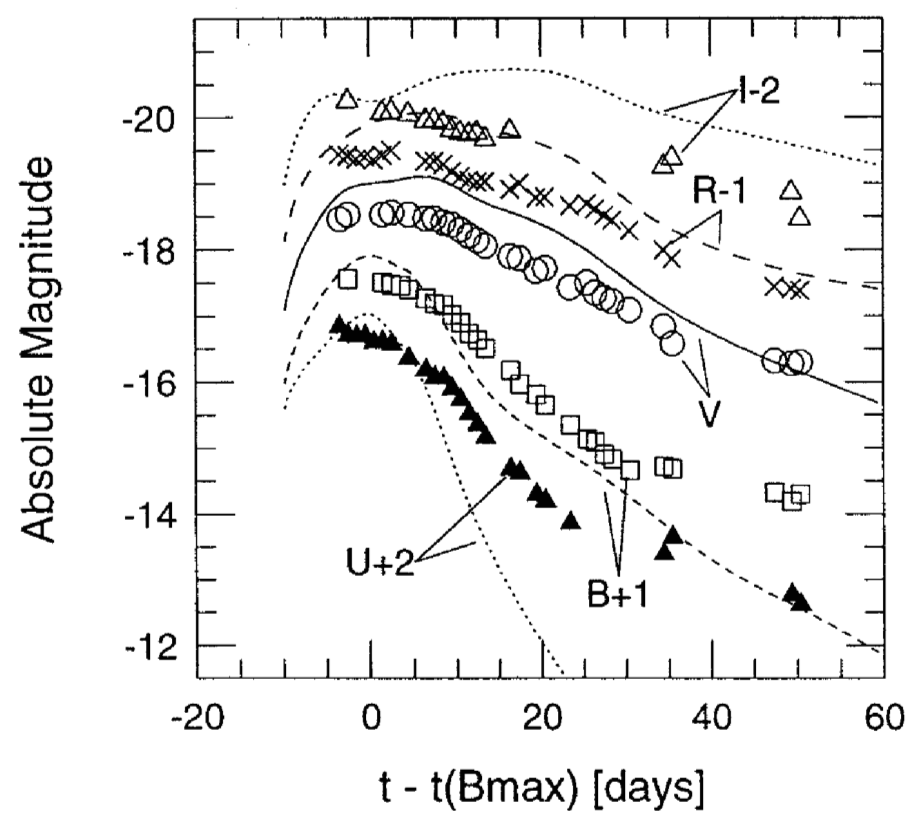

Figure 6. This figure compares UBVRI photometry of SN 1989B (Wells et al. 1994) to the computed light curves of the sub- $M_{c h}$ Model 2. 


\section{RADIATION TRANSPORT IN TYPE IA SUPERNOVAE}

outward from the center, and a diffusing optical wavelength photon is likely to come into resonance with several strong transitions before reaching the surface. In the calculations which led to Figures 7 and 8 it was assumed that all photons absorbed in line transitions were completely thermalized. However, as Figure 2 shows, the most likely fate is reemission at another wavelength. Passing through resonance with a strong line does not necessarily lead to thermalization.

By 28 days, the optical spectrum in both of these models will be dominated by emission lines. The continuum optical depth is not large enough to produce a strong underlying continuum. Instead, the emergent optical spectrum is a pseudo-continuum produced by line photons which are Doppler broadened by repeated scatterings off of free electrons.

What the small optical depths in these two models mean is that departures from LTE are likely to be significant even at maximum light. It also means that the equilibrium radiative diffusion approximation, in which $J_{\nu} \approx B_{\nu}$, is not likely to be very accurate at optical wavelengths, especially in a model like Model 2, and spectrum calculations which invoke the diffusion approximation as a lower boundary condition to an "atmosphere" calculation (e.g. Nugent et al. 1995) will predict inaccurate optical fluxes. Figure 9 compares the frequency integrated mean intensity to the frequency integrated Planck function at the local gas temperature at three times before and after maximum light in Model DD4. By 22 days after explosion the radiation field has become quite dilute, and the assumption of LTE is likely to be in serious error.

\subsection{COMPARISON OF $\kappa_{H}$ AND $\kappa_{R}$}

With the radiation transport solution from EDDINGTON it is possible to compute $\kappa_{H}$, the flux mean opacity, and see how well it compares with the Rosseland mean, $\kappa_{R}$. Figure 10 displays the run of $\kappa_{H}, \kappa_{R}$ and $\kappa_{e}$, which is the electron scattering mass opacity coefficient, for Model DD4, at three different times after explosion. I've defined $\kappa_{R}$ as

$$
\kappa_{R}^{-1}=\frac{\rho \pi t}{a T^{3}} \int_{\nu}^{\infty} \frac{\partial B_{\nu^{\prime}}}{\partial T}\left(\nu^{\prime}\right)^{-1} \exp \left\{-c t \int_{\nu}^{\nu^{\prime}} \frac{\left(k_{\nu^{\prime \prime}}+\sigma_{\nu^{\prime \prime}}\right)}{\nu^{\prime \prime}} d \nu^{\prime \prime}\right\} d \nu^{\prime}
$$

which takes into account expansion (however see the discussion by Blinnikov, this volume). At all times the flux mean opacity is substantially greater than the Rosseland mean, by as much as a factor of 3 at maximum light. The reason these two means are so different is that, at any one place in the ejecta, the radiation field has a slightly higher color temperature than the local gas temperature and, because of the steep wavelength dependence of the line opacity, this small imbalance results in a sizeable 
RONALD G. EASTMAN
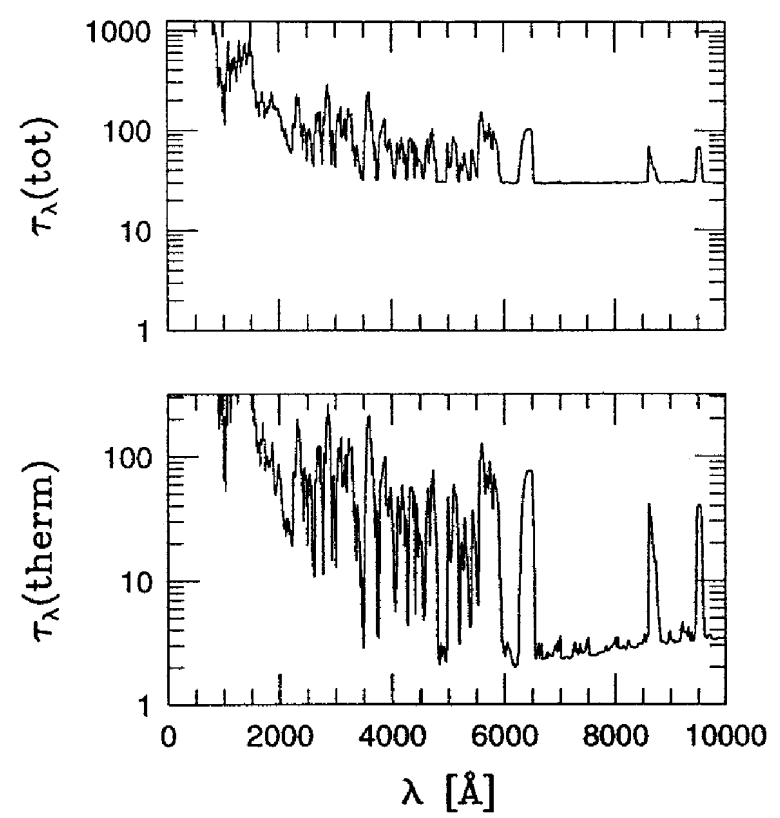

Figure 7. The top panel shows the monochromatic optical depth to the center as measured in the observer frame for Model DD4 at 14 days after explosion. The lower panel shows the thermalization optical depth, also in the observer frame, assuming lines are completely absorptive.

difference between the Rosseland and flux mean. Unfortunately, this brings into question the results of evolutionary calculations which use single temperature $\left(T_{r a d}=T_{g a s}\right)$ Rosseland mean opacities (e.g. Höflich, Muller \& Khokhlov 1993; Khokhlov, Muller \& Höflich 1993; Höflich 1995). A lower mean opacity would tend to make the $M_{c h}$ mass models behave similar to a smaller mass star with a higher mass opacity coefficient.

It is possible that the $\kappa_{R}$ values shown in Figure 10 would be different if, for instance, a line expansion opacity approximation other than that of Eastman \& Pinto (1993) were used, or if more lines were included in the list. The important point is that, as the supernovae expands, density drops and mean free paths grow, the equilibrium radiative diffusion approximation breaks down and $\kappa_{H} \neq \kappa_{R}$. In this case, accurate light curves can only be obtained by solving the full multigroup transport problem.

\section{Old Photons}

Some researchers have put forth the idea that one can reproduce the emergent spectrum of a maximum light SNe Ia by placing an atmosphere on top 

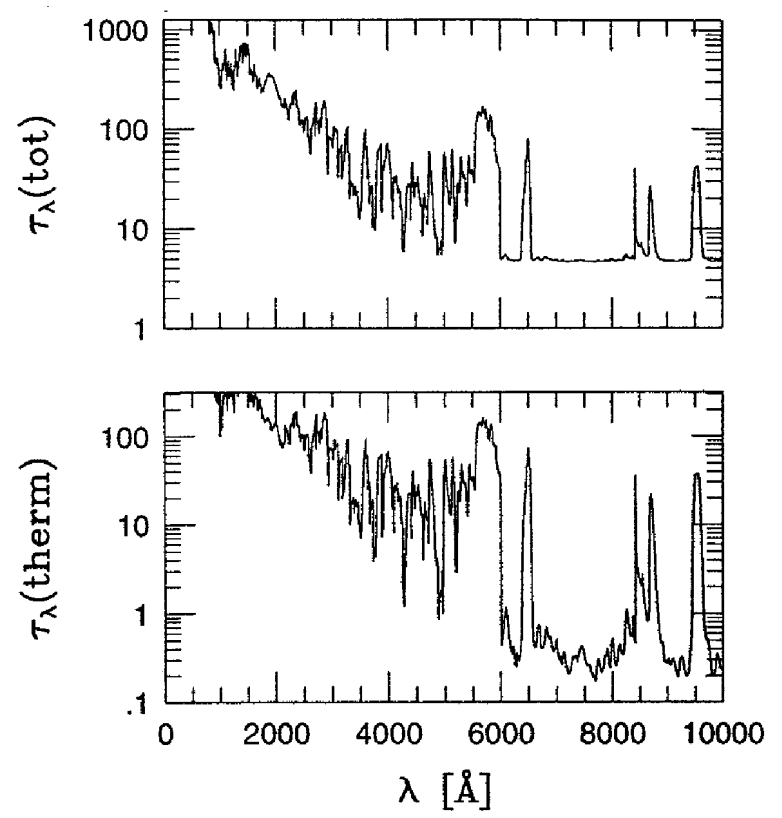

Figure 8. Same as Figure 7, but for Model 2.

of a lower diffusion boundary condition, and that by adjusting the luminosity at the lower boundary to the desired value, it is possible to accurately compute the maximum light thermal structure of the atmosphere and the emergent spectrum. I would like to explain here the reasons why results of such a calculation could be very different from actual maximum light $\mathrm{SNe}$ Ia.

First, as discussed in the proceeding section, the thermalization optical depth at maximum light, even in a $M_{c h}$ model like DD4, is small. Equilibrium radiative diffusion is only accurate when the thermalization optical depth is large $(\tau$ (therm) $>>2$ !).

Second, at maximum light the radiation field is evolving on a rapid time scale. As I will attempt to show here, a significant fraction of the emergent luminosity at maximum light is from energy deposited at significantly earlier times, and only by solving the full time dependent problem can the radiation field and thermal structure be determined.

Pinto and Eastman (1996; see also Pinto, this volume) derive several useful analytic approximations to the problem of equilibrium radiative diffusion in a variable density, variable deposition, variable opacity, freely expanding sphere, with realistic surface boundary conditions. Here I will consider only the simplest possible case: that of constant density (in space), 
RONALD G. EASTMAN

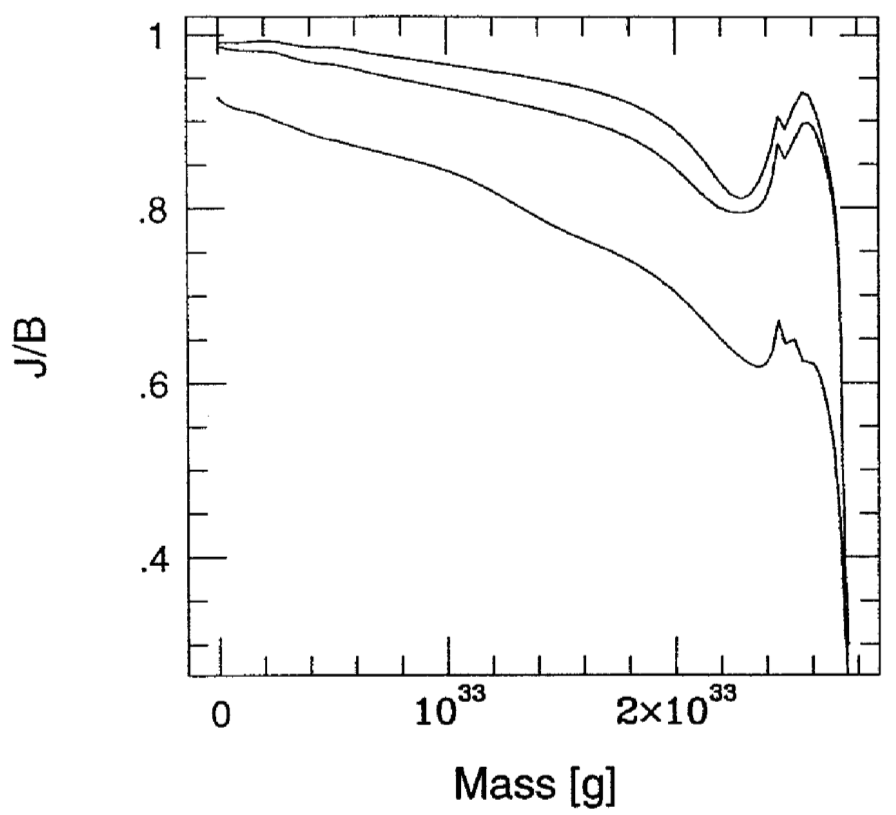

Figure 9. Ratio of frequency integrated mean intensity to the frequency integrated Planck function at the local gas temperature, versus Lagrangian mass coordinate, in Model DD4, at (from top to bottom) $t=11.2, t=13.8$ and $t=22$ days after explosion.

constant mass opacity coefficient, and radiative zero boundary condition. This would be the same problem solved by Arnett (1982), except that I will allow the $\gamma$-ray deposition function to vary spatially in a semi-realistic way.

Consider the case where with deposition function is given by $\dot{S}(r, t)=$ $\dot{S}(t) \times \sigma(r)$, with

$$
\sigma(r)=X_{56} \times \begin{cases}1 & \text { if } r /\left(R_{0}+v_{s c} t\right) \leq x_{0} \\ 0 & \text { otherwise }\end{cases}
$$

where $R_{0}$ is the outer radius at time $t=0, v_{s c}$ is its expansion velocity, $X_{56}$ is the ${ }^{56} \mathrm{Ni}$ mass fraction, and

$$
\begin{aligned}
\dot{S}(t)= & \left(\dot{S}_{\mathrm{ni}} \exp \left[-t / t_{\mathrm{ni}}\right]+\left(\dot{S}_{\mathrm{co}} \exp \left[-t / t_{\mathrm{co}}\right]-\dot{S}_{\mathrm{ni}} \exp \left[-t / t_{\mathrm{ni}}\right]\right)\right) \times \\
& \left(1-f_{\text {esc }}(t)\right) \times\left(1-f_{e^{+}}(t)\right)+ \\
& \left(\dot{S}_{\mathrm{co}} \exp \left[-t / t_{\mathrm{co}}\right]-\dot{S}_{\mathrm{ni}} \exp \left[-t / t_{\mathrm{ni}}\right]\right) \times f_{e^{+}}
\end{aligned}
$$

is the deposition rate in ergs $\mathrm{s}^{-1} \mathrm{~g}^{-1}$ of ${ }^{56} \mathrm{Ni}$. The various constants in eqn (9) are $\dot{S}_{\mathrm{ni}}=3.9 \times 10^{10} \mathrm{ergs} \mathrm{g}^{-1} \mathrm{~s}^{-1}, \dot{S}_{\mathrm{co}}=7.03 \times 10^{9} \mathrm{ergs} \mathrm{g}^{-1} \mathrm{~s}^{-1}$, 


\section{RADIATION TRANSPORT IN TYPE IA SUPERNOVAE}

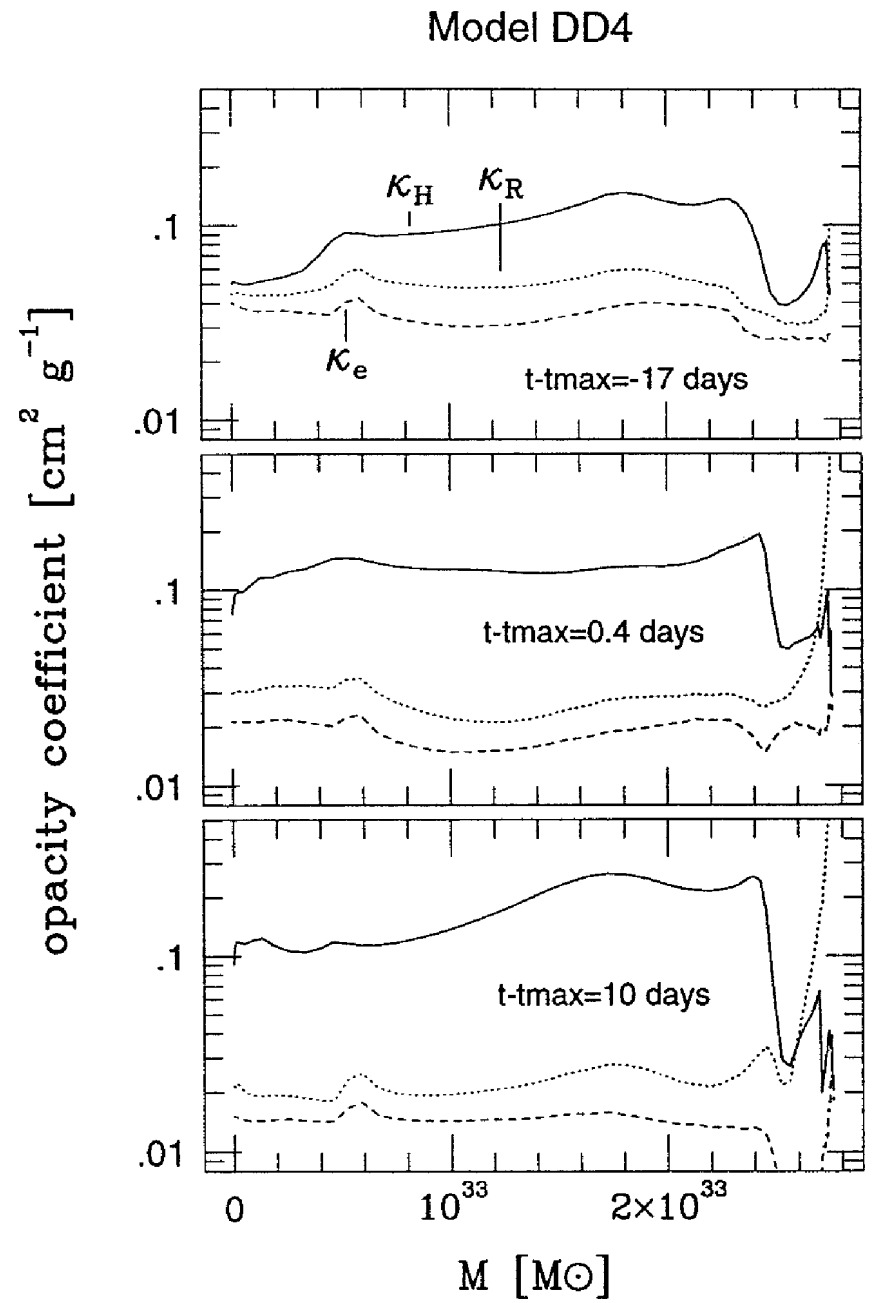

Figure 10. Comparison of flux mean $\left(\kappa_{H}\right)$, Rosseland mean $\left(\kappa_{R}\right)$ and electron scattering $\left(\kappa_{e}\right)$ mass opacity coefficients in Model DD4 versus Lagrangian mass coordinate at three times before and after bolometric maximum.

$t_{\mathrm{ni}}=8.8$ days and $t_{\mathrm{co}}=111.5$ days. Also, $f_{e^{+}}=0.0339$ is the fraction of ${ }^{56} \mathrm{Co}$ decay energy in the form of positron kinetic energy (which I assume is locally processed), and $f_{e s c}(t)$ is the $\gamma$-ray escape probability, which I'll take to be given by

$$
f_{\text {esc }}(t)=\exp \left(-\kappa_{\gamma} \rho_{0} R_{0}\left(1+\frac{t}{t_{s c}}\right)^{-2}\right)
$$

where $t_{s c}=R_{0} / v_{s c}, \rho_{0}$ is the mass density at time $t=0$, and $\kappa_{\gamma}$ is the 
RONALD G. EASTMAN

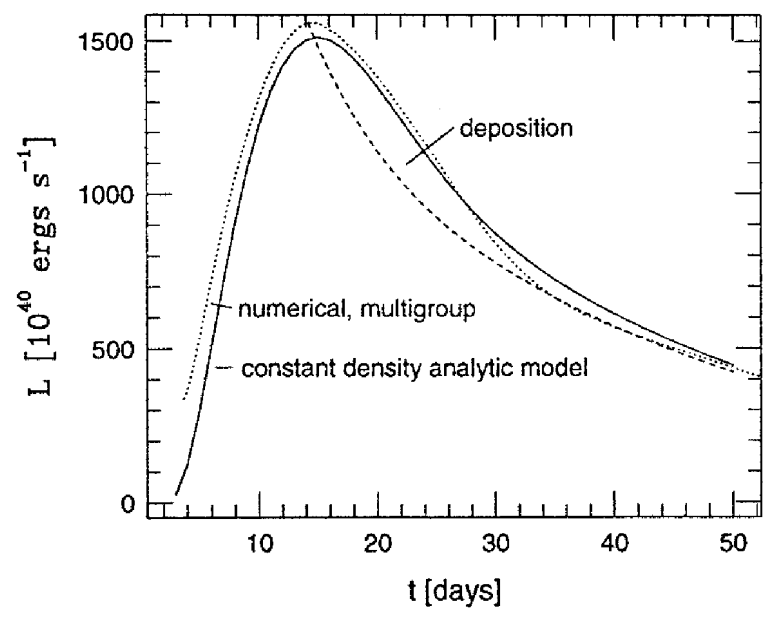

Figure 11. This figure compares the bolometric light curve of Model DD4 computed by a multi-group transport calculation with EDDINGTON, to that predicted by eqn (11). Both the analytic model and Model DD4 have $M_{\text {tot }}=1.386 \mathrm{M}_{\odot}, E=1.11 \times 10^{51} \mathrm{ergs}$, and $M_{56}=0.625 \mathrm{M}_{\odot}$. Additionally, it was assumed for the analytic model that $R_{0}=10^{9} \mathrm{~cm}$, $\kappa_{0}=0.1 \mathrm{~cm}^{2} \mathrm{~g}^{-1} \kappa_{\gamma}=0.05 \mathrm{~cm}^{2} \mathrm{~g}^{-1}$, and $X_{56}=1$ for $r \leq\left(M_{56} / M_{t o t}\right)^{1 / 3}\left(R_{0}+v_{s c} t\right)$, and zero otherwise.

$\gamma$-ray mass opacity coefficient.

For these assumptions, the emergent luminosity is given by

$$
L(t)=\frac{4 \sqrt{2} \pi^{2} R_{0} c}{3 \kappa_{0}} \sum_{n=1}^{\infty} n(-1)^{n+1} A_{n}\left(x_{0}\right) \int_{0}^{t} \dot{S}\left(t^{\prime}\right) \phi_{n}\left(t ; t^{\prime}\right)\left(1+\frac{t^{\prime}}{t_{s c}}\right) d t^{\prime}
$$

where $\kappa_{0}$ is the thermal photon mass opacity coefficient,

$$
A_{n}=X_{56} \frac{\sqrt{2}}{(n \pi)^{2}}\left(\sin \left(n \pi x_{0}\right)-n \pi x_{0} \cos \left(n \pi x_{0}\right)\right)
$$

and

$$
\phi_{n}\left(t ; t^{\prime}\right)=\exp \left(-\frac{(n \pi)^{2}}{t_{d}}\left(1+\frac{t^{\prime}}{t_{s c}}\right)\left(t-t^{\prime}+\frac{\left(t-t^{\prime}\right)^{2}}{2\left(t^{\prime}+t_{s c}\right)}\right)\right)
$$

In this equation, $t_{d} \equiv 3 R_{0}^{2} \rho_{0} \kappa_{0} / c$ is the initial diffusion time.

The accuracy of this approximation is shown by Figure 1.1, which compares the EDDINGTON light curve of Model DD4 with $L(t)$ predicted by eqn (11), and parameters appropriate to DD4. The mass opacity coefficient was taken as $\kappa_{0}=0.1$, which is consistent with the results for $\kappa_{H}$ shown in Figure 10. Considering the simplicity of the model, it does a very good job at reproducing the bolometric luminosity of Model DD4. 


\section{RADIATION TRANSPORT IN TYPE IA SUPERNOVAE}

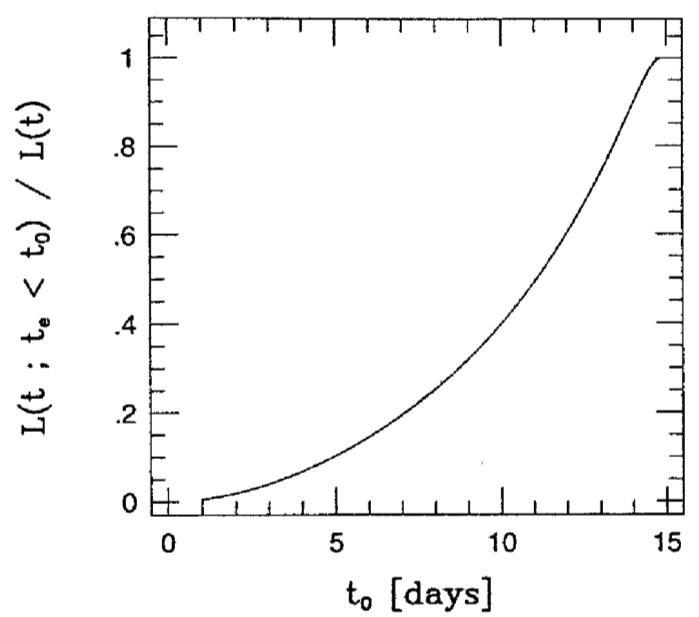

Figure 12. This figure shows the fractional contribution to the bolometric luminosity at $t=15$ days from energy deposited at times earlier than $t_{0}$, for the analytic model. Parameters for the analytic model are those appropriate to Model DD4, as described in the caption to Figure 11 and in the text.

With eqn (11), we can determine the fractional contribution to the instantaneous luminosity at time $t$ from deposition at times earlier than time $t_{0}$ :

$L\left(t ; t_{e}<t_{0}\right)=\frac{4 \sqrt{2} \pi^{2} R_{0} c}{3 \kappa_{0}} \sum_{n=1}^{\infty} n(-1)^{n+1} A_{n}\left(x_{0}\right) \int_{0}^{t_{0}} \dot{S}\left(t^{\prime}\right) \phi_{n}\left(t ; t^{\prime}\right)\left(1+\frac{t^{\prime}}{t_{s c}}\right) d t^{\prime}$

Figure 12 displays $L\left(t ; t_{e}<t_{0}\right) / L(t)$ at $t=15$ days versus $t_{0}$ for the same model parameters used to compare with DD4 in Figure 11. What this figure shows is that at 15 days, 50 percent of the emergent luminosity is from energy which was deposited earlier than 11 days after explosion, 25 percent from deposition earlier than 8 days after explosion. At 15 days, 7 days earlier is a significant fraction of the age of the supernova! It follows that steady state approximations are likely to be highly inaccurate, and the ejecta thermal structure to be much more complicated than would be obtained by, for instance, placing a diffusion boundary condition at the bottom of an atmosphere and assuming constant (with depth) luminosity, or by balancing the instantaneous radioactive decay heating rate against thermal emission losses. It is only by solving the fully time dependent transport problem that the thermal structure can be accurately determined, and definitive statements made about the extent to which a given explosion model agrees or does not agree with Type Ia supernova observations. 
I would like to thank Ramon Canal and Pilar Ruiz-Lapuente for their hard efforts at putting together such a productive and enjoyable meeting.

This work was performed under the auspices of the U.S. Department of Energy by the University of California, Lawrence Livermore National Laboratory under Contract No. W-7405-Eng-48.

\section{References}

Arnett, W. D. (1982), ApJ, 253, 785.

Bartunov, O. S., Blinnikov, S. I., Pavlyuk, N. N. \& Tsvetkov, D. Yu. (1994) $A \& A, \mathbf{2 8 1}, \mathrm{L} 53$.

Blinnikov, S. I. \& Bartunov, O. S. (1993) A\&A, 273, 106.

Blinnikov, S. I., Bartunov, O. S., Popolitov, V. A., Eastman, R. G. \& Woosley, S. E. (1996), in preparation for ApJ.

Eastman, R. G. \& Pinto, P. A. (1993), ApJ, 412, 731.

Harkness, R. P. (1991) in SN 1987A and Other Supernovae, eds. J. Danziger and K. Kjär (ESO: Garching), 447.

Höflich, P., Muller, E. \& Khokhlov, A. (1993), A\&A, 268, 570.

Höflich, P. (1995), ApJ, 443, 89.

Karp, A., Lasher, G., Chan, K. L. \& Salpeter, E. E. (1977), ApJ, 214, 161.

Khokhlov, A., Muller, E. \& Hoflich, P. (1993)), A\&A, 270, 223.

Kurucz, R. L., (1991), in Stellar Atmospheres: Beyond Classical Models, eds. L. Crivellari, I. Hubeny \& D.G. Hummer (Kluwer:Dordrecht) 441.

Montes, M.J. \& Wagoner, R. V. (1995), ApJ, 445, 828.

Nomoto, K., Thielemann, F. K., \& Yokoi, K. (1984) ApJ 286, 644.

Nugent, P., Baron, E., Hauschildt, P. H. \& Branch, D. (1995), ApJ, 441, L33.

Phillips, M. M., Wells, L.A., Suntzeff, N.B., Hamuy, M., Leibundgut, B., Kirshner, R. P, \& Foltz, C. B. (1992), AJ, 103, 1632.

Phillips, M. M. (1993) ApJ Lett, 413, 105.

Pinto, P. A. \& Eastman, R. G. (1996) in preparation.

Suntzeff, N. B. (1995) IAU colloquium 145: Supernovae and Supernova Remnants, ed. R. McCray, (Cambridge: Cambridge University Press), in press.

Wells, L. A., Phillips, M. M. Suntzeff, N. B., Heathcote, S. R. and others (1994), AJ, 108, 2233. 


\section{RADIATION TRANSPORT IN TYPE IA SUPERNOVAE}

Wagoner, R.V., Perez, C.A. \& Vasu, M. (1991) ApJ, 377, 639.

Wheeler, J. C., Swartz, D. A. \& Harkness, R. A. (1991) Phy. Rep., 227, 113.

Woosley, S. E. \& Weaver, T. A. (1991), in Les Houches, Session LIV, eds. J. Audouze, S. Bludman, R. Mochkovitch and J. Zinn-Justin (Elsevier Science Publishers).

Woosley, S. E. \& Weaver, T. A. (1994), ApJ 423, 371.

Woosley, S. E., Eastman, R. G., Weaver, T. A. \& Pinto, P. A. (1994), ApJ, 429,300 . 